\title{
Feature-based Indoor Navigation using Augmented Reality
}

\author{
Sebastian Kasprzak, Andreas Komninos, Peter Barrie \\ School of Engineering \& Computing \\ Glasgow Caledonian University \\ Glasgow, United Kingdom \\ sebastian@kasprzak.co.uk, [andreas.komninos, peter.barrie]@gcu.ac.uk
}

\begin{abstract}
We present a prototype for indoor navigation using Augmented Reality that uses interior features to determine the user's location and provide navigation instructions. We test our prototype in a simulated physical shopping mall environment and find that $A R$-based navigation can provide usability advantages in indoor locations, particularly where targets are located on different floors. We conclude by recommending further work in presenting interior navigation instructions using AR.
\end{abstract}

\section{Keywords-Augmented Reality, Decision Support Systems}

\section{INTRODUCTION}

Navigation has been an important subject of research for many years. People have always been interested in developing devices, technologies and applications that help them find their location, and aid in navigating to destinations. According to a recent study performed on mobile phone users [1], around $95 \%$ of users who own a Smartphone device used a mapping application at least once, while $80 \%$ of users used it more than 10 times during the period of study, which indicates that maps are in everyday use for most of Smartphone users. Navigation requires knowledge of current position. The availability of cheap, globally available technology supporting current positioning through GPS has been a major driving force in the popularity of navigation applications for mobile devices. Ever since GPS became popular on a global scale, it has always been expected that a similar global solution for indoor navigations will be available sooner rather than later. GPS poses wellknown problems if it is used indoors, due to its requirements for a clear line of sight with earth-orbiting satellites for precise positioning. Current technology gives no straightforward answer to a low-cost, universal indoor mobile navigation system which requires no installation or dedicated infrastructure, as indoor navigation systems are often based on bespoke technologies that use ultrasonic or radio frequencies and infrared.

\section{INDOOR POSITIONING AND NAVIGATION WITH AUGMENTED REALITY}

\section{A. Indoor positioning}

Mulloni et al. [8] distinguish the differences between continuous localization and discrete localization. Continuous localization informs users of their current location at all times, and a good example of it would be a GPS car navigation system. For discrete localization it is required to first find and then recognise some type of marker or landmark. A device's current location can be updated only after such a discrete marker is scanned. Indoor positioning literature covers a range of technologies used to achieve continuous indoor localization (and thus navigation), such as infrared technology [3], WLAN's and signal strengths [4], dead reckoning [6] and more. Discrete positioning has been attempted in the past using techniques such as image analysis and comparison with dedicated databases [14] or the web [15]. Of course the cost of such a technique resides in the requirement for constructing large databases or using natural language processing techniques to filter out web results. While interesting, these techniques are probably not well suited for indoor navigation since building an indoor image database requires the capture of much more information than just the facades of buildings (as in [14]). Additionally, the web contains many more exterior images of landmarks than interiors.

Fiducial markers [7] [8] [9] offer an attractive alternative to discrete indoor positioning, as these can often be installed with very little cost. These specially constructed markers are optically recognisable by a camera-equipped device and as such can help with both positioning and the display of AR visual cues for navigation, on top of the markers. While several augmented reality projects have used marker-based localization little has been done towards research on continuous localization using Augmented Reality. Klopshitz et al. [5], by trying to achieve continuous localization, used a large quantity of fiducial markers located at every location within the whole building. Such an approach assumes that the camera that is capturing the fiducial markers will always have a fiducial marker visible to enable localization. The authors describe this method as scalable, but there is a requirement for extensive implementation and calibration, which could become costly on large scale. Mulloni et al.'s work [7] tries to achieve dead reckoning by the use of a step counter to enable continuous localization, though this technique is known to be susceptible to high error rates. Others such as [16] and [17] have used inertial navigation techniques with success, though their work requires specially mounted wearable sensors which render such a system impractical for daily use.

\section{B. Presenting Navigation Instructions}

While some of the projects in indoor AR navigation have delivered very accurate indoor navigation systems, all have faced one major disadvantage, which is typically a requirement to wear a computing device and some sort of head mounted sensor (e.g. Kim et al. [10]). This has always 
constrained indoor navigation and augmented reality projects to be usable principally in lab environments, being impractical in everyday use. Kray et al. [18] suggests, after investigating spoken and written navigation instructions, as well as 2D sketches and 2/3D maps that "Route sketches are a good compromise in terms of resource consumption and dependency on positional information. Since only a few very simple forms have to be drawn, they are well adapted to the small display size of a mobile device. They are easily understood but do offer little context, and providing additional context comes at the cost of increased complexity." They conclude that fully detailed 2D or 3D maps are not necessary when indoor positioning works precisely (such as in the case of a fiducial marker system) and as such, a simple sketch-based system (e.g. a few arrows) could be efficient and appropriate for mobile devices.

Using a visual arrow motif to indicate direction is a wellknown and commonly used methodology for giving directions. An early example by Wagner [11] showed how AR might be used to present navigation instructions, though this work focused on the technical implementation details and not the user behaviour with such a system. Both Mulloni [7] and Merico [6] use a system of arrows to demonstrate the path to be taken by user. Mulloni et al., 2011 [7] take an approach of directing users only towards the next "info point", instead of showing full path to the destination, to limit the number of directions given to user at once. Presenting users with small amount of directions at every occasion was shown to have the potential to lower down the number of errors made while navigating. Kim et al. [10] in their application also used arrows to guide users on the direction to follow. In addition to that, a small map showing current location was also visible, which aimed to improve a user's impression of their orientation within the building.

Müller et al [12] take a different approach by using building maps placed on indoor corridor walls as fiducial markers upon which the user's route is superimposed via a handheld AR device. Their system does not provide any indication on whether the user needs to turn left or right to find their target but simply shows the path. The researchers found that AR users performed significantly slower but with fewer errors than participants who could not augment the building maps. Brush et al. [2] introduced an activity-based navigation approach, in which users are prompted to perform a list of activities, such as "take the stairs up one floor then walk north 50 steps" to get to their destination. Brush et al. also state that activity-based navigation is "an interesting alternative to map based navigation [...] or indoor localization systems because it does not require that a map be provided or constructed" [2]. Their research concludes that using a step-counter was not particularly helpful while performing activity-based navigation. Brush's research participants also indicated that the step counter used on its own, or in conjunction with pictures of an environment, caused significant frustration while performing activities. Use of a step counter together with a compass, or verbal activity-based navigation, offered better results in Brush's research.

\section{USING BUILDING FEATURES AS TARGET MARKERS}

As the literature indicates, using a markerless approach is either significantly error-prone (dead-reckoning) or requires a significant infrastructure, which increases the implementation and maintenance costs. When considering the way people actually navigate or give instructions, it is easy to realise that in most indoor locations, there exist clear "markers" which are purposely constructed to offer localization and instructions (e.g. signs) or "features" which are used by humans as landmarks of reference (e.g. "turn right after the elevator" or "half-way down this corridor"). One might consider "markers" to be a subclass of a building's "features". As such we wondered whether an application that could detect such "features" could work well in providing both localization and navigation information using augmented reality on a handheld device.

Our initial approach was to build a graph to represent a building's internal structure, using human-readable signs as nodes and applying a path finding algorithm adapted from Dijkstra's well known algorithm, to offer navigation instructions that include concepts such as "behind", "turn around" and "up/down the stairs". We used Qualcomm's Vuforia SDK for Android to allow the optical recognition of door signs in an office building (university) and designed a simple interface where the user could either type in their current location and destination, or have the application automatically recognise the door sign using optical scanning (Fig. 3).

The Vuforia SDK is not an optical character recognition (OCR) system but works on the principle of pattern matching. An application developer can upload "trackable" images to the Vuforia website, where an in-built algorithm extracts feature-points from each image after turning it into grayscale. The feature-point data can then be embedded into the application so that it can recognise and track the objects shown by the image. Upon testing, we found that the system had trouble distinguishing between the door signs on several occasions, since the SDK depends on identifying multiple "feature points" on the images and the door signs were found to be too similar (the signs contained an office number and some text) to each other to provide accurate results (Fig. 1).

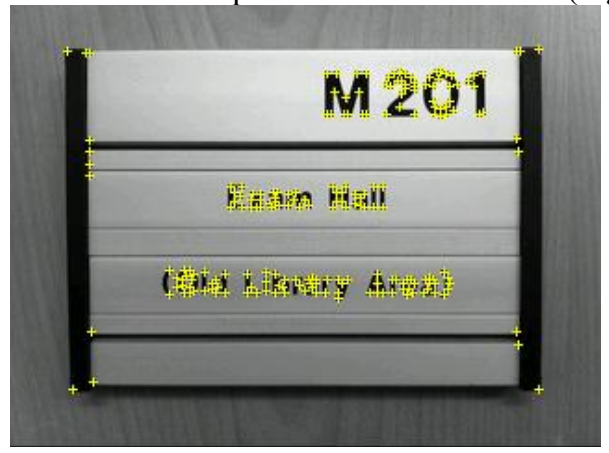

Figure 1. Visible features of a door sign via Qualcomm Vuforia SDK 

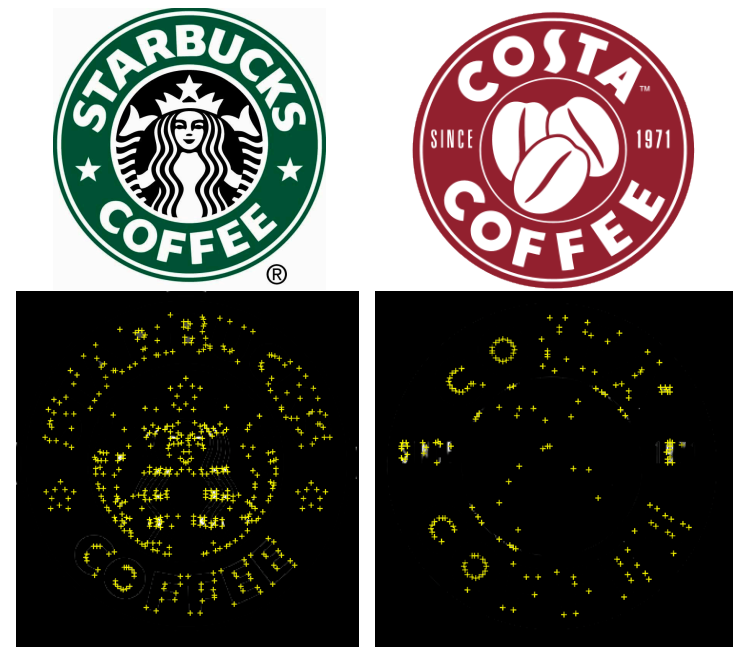

Figure 2. Two optically "similar" logos from actual businesses within a mall. After feature detection, it can be seen that it is easy for a system to distinguish between the two

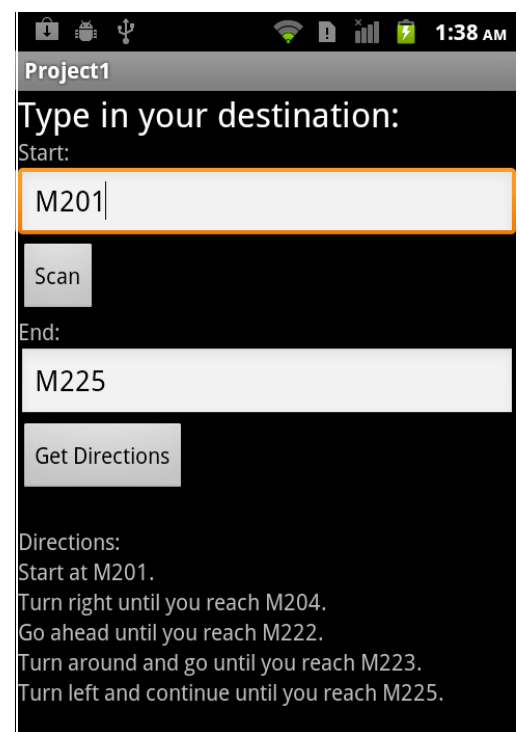

Figure 3. Initial prototype

We then considered applying the system in a different type of environment, where more discrete "features" or "landmarks" could be found. A shopping mall was selected for this purpose, since the main navigation landmarks to be found there are the different shops, which all have very distinct and unique logos that are clearly displayed on the shop front. We examined the logos of all stores in a large shopping mall and found that the SDK's recognition performance in distinguishing each logo was consistently high, even though the Vuforia SDK trackable analysis website gave low suitability ratings for many of the logos tested (Fig. 2).

\section{FIELD TRIAL OF THE SYSTEM}

\section{A. Adaptation of the application}

Prior to testing our system, we modified the user interface so that the user is presented with a list of all shops in the mall. The user selects an appropriate destination from the list and is immediately transferred to the viewfinder screen, which can be used to scan targets and view navigation information. Every time a target is successfully recognised, the algorithm recalculates the route and provides instructions to the user. So that the user does not get overloaded with information, we present only the next two steps of the route. The user can then select to either scan every other shop to get an instruction update, or to keep going towards the general direction of their destination and randomly scan a target if they feel lost.

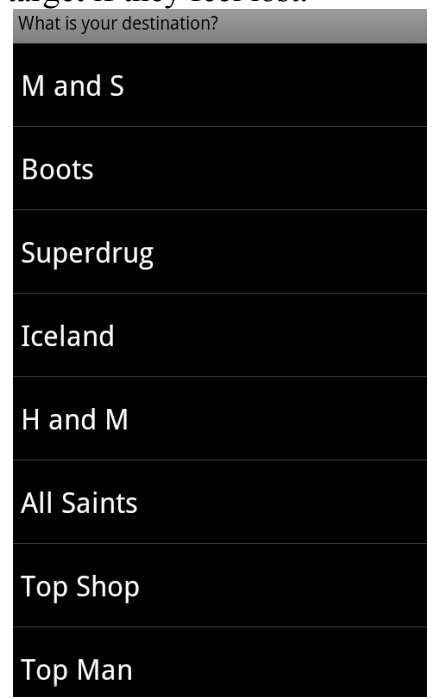

Figure 4. Final Pathfinder prototype destination selection screen

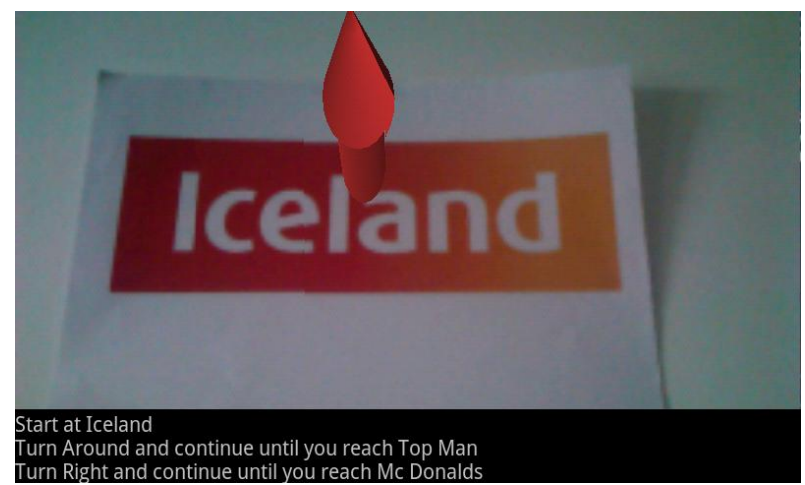

Figure 5. The final prototype Augmented Reality directions (arrow) and accompanying text directions 


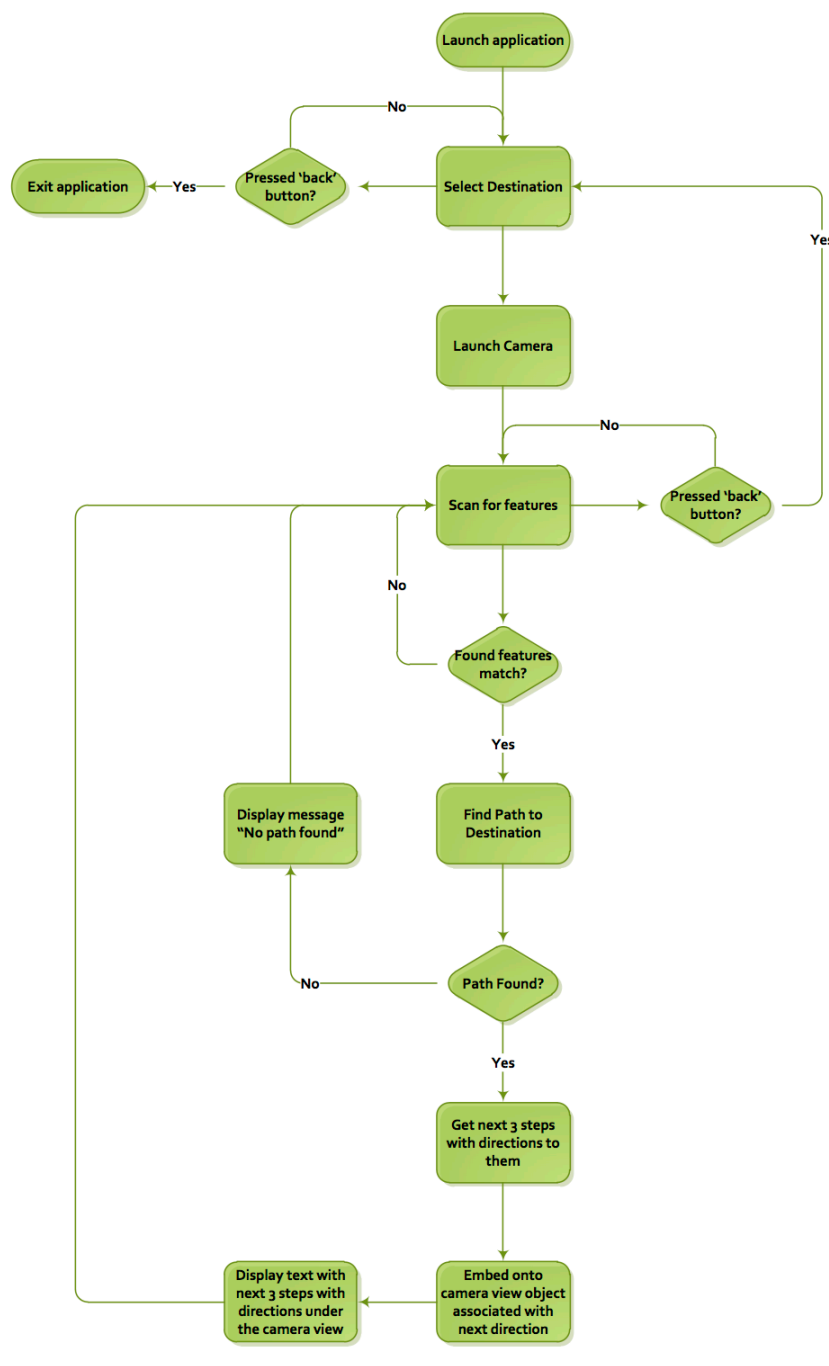

Figure 6. The Pathfinder system application logic

\section{B. Participants}

Our aim was to compare the usefulness of our Augmented Reality application in giving directions compared to the use of a paper map, as often used in complex buildings as shopping malls. To determine our application's effectiveness, we gave participants three navigation tasks that related to locating three targets (shops) in an unfamiliar indoors environment. We recruited 20 participants, which we split into two equal size groups and gave them the same navigation tasks to carry out using our AR application (Group 1) or a paper map (Group 2). Due to some participants not turning up for the experiment, we ended up with 9 participants in Group 1 (ages 19-30, mean = 22.7, stdev $=3.24,5$ females) and 8 participants in Group 2 (ages 20-27, mean $=23.0$, stdev $=2.87,4$ females). None of the participants were familiar with AR based systems and all indicated familiarity with using printed maps. Each participant was given a few minutes to familiarise themselves with the use of the application, or the paper map, depending on their group before the start of the tasks.

\section{Environment}

While we hoped initially to carry out our trial in an actual shopping mall environment, we encountered two major obstacles. First, photographing storefronts from our city's two major shopping malls, in order to use the images as trackables, was not permitted due to business operational rules. Sadly, even though we approached the shopping mall management and explained our purpose, we were strictly prohibited from photographing or using the interior of the shopping mall for our experiment. Secondly, the participants that we had access to were likely to be rather familiar with these shopping mall environments, as these were close to the university and probably frequently visited by the students.

To proceed with our study, we decided use the interior of a university building to simulate the "shopping mall". The chosen building was an office building for administration services, which the students we used as participants were unfamiliar with as access to the entire building is normally restricted to university staff only. We printed out 53 different shop logos on A3 size paper and positioned them into the building environment over two floors. Fig. 5 shows the positioning of the store logos over the two floors and Fig. 6 depicts aspects of the actual testing environment. The final testing environment is not without compromise or limitation. For example, the shop logos are much smaller than they would be in an actual shopping mall. This prevents the user from detecting targets visually from a reasonable distance (e.g. more than 20 meters) as they might be able to do in an actual mall, thus rendering users more reliant on the Pathfinder or paper map aids. Additionally, the shop logos are positioned at eyesight level and not at a height typical of most shop logos (e.g. above doorways). The purpose of this positioning was to prevent excessive "skewing" and distance from interfering with the Vuforia SDK's ability to correctly detect targets in the camera frame.

Our purpose here is not to investigate the performance of the SDK but the high-level task of navigation through AR. Additionally, we are not interested in limiting our study to shopping malls per se, but to focus on the usability of AR systems in a generic indoor environment (e.g. instead of shop logos someone might be tracking door signage or directional signs, such as in a hospital). Thus we felt that the chosen environment setup was a reasonable for our purpose.
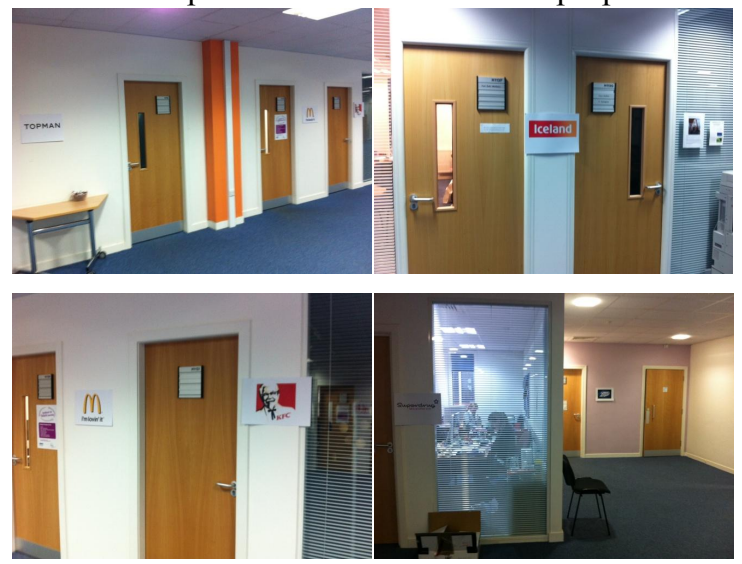

Figure 7. The trial environment 

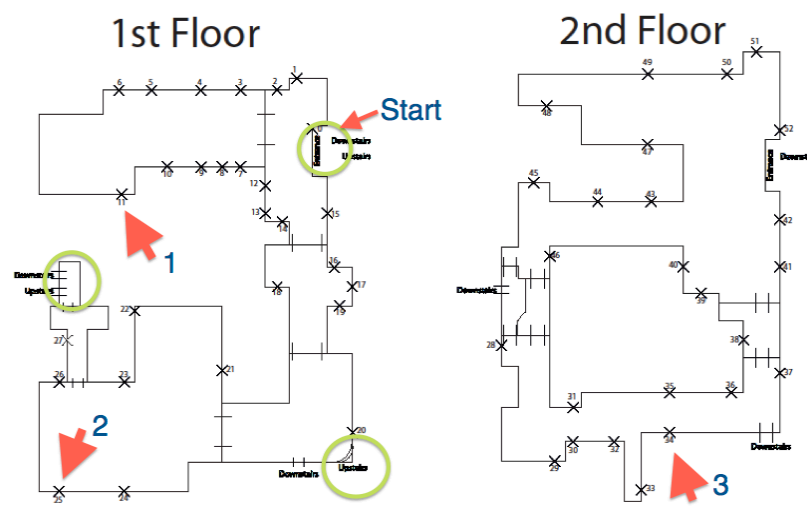

Figure 8. The trial environment targets (crosses indicate the location of features)

\section{Tasks}

All participants started at the same location and were required to sequentially find three targets (shops), using each target as a starting point for the next one. The green circles in Fig. 5 denote the staircases that connect the two floors. A researcher followed the participants, in order to take notes on their behavior. The researcher did not interfere with the participants in any sort of manner and the participants were told that they could not address the researcher. Each participant was timed from the starting point until the moment they reached the final target. The researcher following the participants annotated each participant's path on a copy of the floor map and noted various behavioral events and times as they took place. The events noted for both groups were pauses (where the participant stopped and looked around to consider their path) and wrong turns taken (turns that led to a direction other than the one required to reach the target). For Group 1, the researcher also noted scanning events (i.e. use of the application) and whether each scan had been successful (i.e. whether the trackable was recognized by the device). At the end of each run, every participant completed a short questionnaire to obtain subjective evaluation data from their experience.

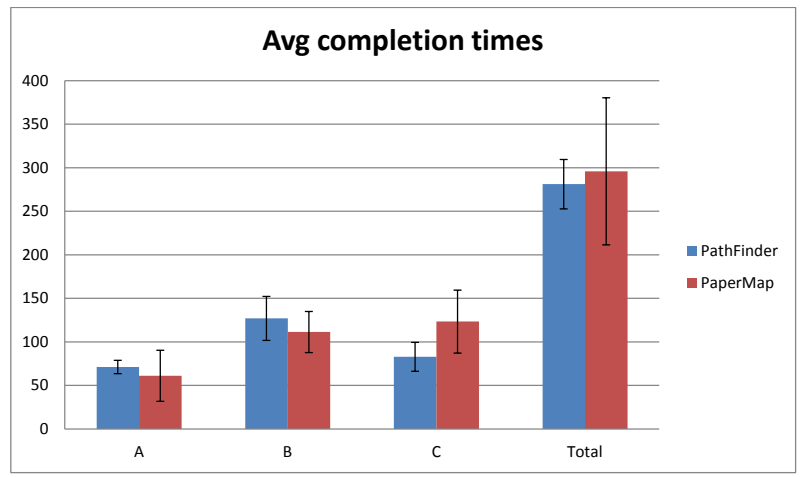

Figure 9. Completion time performance

\section{OUTCOMES AND ANALYSIS}

\section{A. Task Completion times}

It is important to note that task completion rate was at $100 \%$, as all participants from all groups managed to find all destinations. As shown in Fig. 8, the start point and destinations A and B are all on 1st floor of the building. On average, participants using paper maps were 10 seconds quicker in getting to destination A. Similar results are achieved when measuring time performance in getting to destination B. Participants were on average 16 seconds quicker using paper maps. The performance of paper map participants changed for destination $\mathrm{C}$, which is placed on the 2 nd floor of the building. The difference in performance between two groups was 40 seconds on average in favour of the application users.

Getting to destinations A and B was fairly easy for both groups. Paper map users mostly were able to determine the whole route to destination A with just one look at the map. Directions given by the application only informed the participant of the next 2 steps to take, and even if the route was very simple, application users still had to scan for new shops to establish where they should go next. These frequent scans were consuming a lot of their time and were slowing them down. For destinations $\mathrm{B}$ and $\mathrm{C}$, which involved a more complex route to be taken, paper map users were often confused initially at locating the target. Participants also made more errors and had to pause to look at the map several times. Particularly for destination $\mathrm{C}$, application users were given instant directions on which way to go to the nearest staircase, which led to the destination, while paper map participants were often confused about locating the target and finding an optimal path. Taking into consideration total times for completing all three tasks, participants using the application were on average 15 seconds quicker than those using paper maps.

\section{B. Wrong turns and pauses}

Overall, while examining the two charts in Figs. 11 and 12 , it is clear to see that participants who were using paper maps made more errors than participants who were using the application. The clear indication about the direction to follow given by the application helped in keeping these values down; if participants were unsure where to proceed next, they could simply approach the next visible feature to scan its logo to obtain directions on where to go next.

With paper maps, when participants got lost and were unsure on how to proceed, they had to stop to examine the paper map to find their way to destination. Paper map participants also often missed turns, especially when going upstairs to reach destination C. Almost every participant upon going upstairs made a wrong turn by going straight ahead, instead of immediately turning left. Examples of paper map participant behaviour from two participants are depicted in Fig. 10. 
TABLE I.

TASK COMPLETION TIMES FOR GROUP 1

\begin{tabular}{|c|c|c|c|c|}
\hline \multicolumn{5}{|c|}{ Group 1 - Pathfinder system task completion times (seconds) } \\
\hline ID & $\mathrm{A}$ & $\mathrm{B}$ & $\mathrm{C}$ & Total \\
\hline 1 & 67.00 & 102.00 & 71.00 & 240.00 \\
\hline 2 & 65.00 & 143.00 & 86.00 & 294.00 \\
\hline 3 & 83.00 & 124.00 & 69.00 & 276.00 \\
\hline 4 & 71.00 & 179.00 & 93.00 & 343.00 \\
\hline 5 & 77.00 & 141.00 & 73.00 & 291.00 \\
\hline 6 & 69.00 & 97.00 & 116.00 & 282.00 \\
\hline 11 & 72.00 & 129.00 & 76.00 & 277.00 \\
\hline 12 & 58.00 & 108.00 & 97.00 & 263.00 \\
\hline 13 & 79.00 & 120.00 & 65.00 & 264.00 \\
\hline Mean & 71.22 & 127.00 & 82.89 & 281.11 \\
\hline St. Dev & 7.66 & 25.26 & 16.61 & 28.39 \\
\hline Min & 58.00 & 97.00 & 65.00 & 240.00 \\
\hline Max & 83.00 & 179.00 & 116.00 & 343.00 \\
\hline
\end{tabular}

TABLE II. TASK COMPLETION TIMES FOR GROUP 2

\begin{tabular}{c|llll}
\hline \multicolumn{5}{c}{ Group } \\
\hline Participant & A & B & C & Total \\
7 & 28.00 & 77.00 & 68.00 & 173.00 \\
8 & 28.00 & 95.00 & 80.00 & 203.00 \\
9 & 61.00 & 132.00 & 144.00 & 337.00 \\
10 & 94.00 & 111.00 & 130.00 & 335.00 \\
14 & 76.00 & 123.00 & 157.00 & 356.00 \\
15 & 43.00 & 102.00 & 135.00 & 280.00 \\
16 & 107.00 & 152.00 & 169.00 & 428.00 \\
17 & 52.00 & 99.00 & 104.00 & 255.00 \\
\hline Mean & $\mathbf{6 1 . 1 3}$ & $\mathbf{1 1 1 . 3 8}$ & $\mathbf{1 2 3 . 3 8}$ & $\mathbf{2 9 5 . 8 8}$ \\
St. Dev & 29.31 & 23.61 & 36.13 & 84.47 \\
Min & 28.00 & 77.00 & 68.00 & 173.00 \\
Max & 107.00 & 152.00 & 169.00 & 428.00 \\
\hline
\end{tabular}
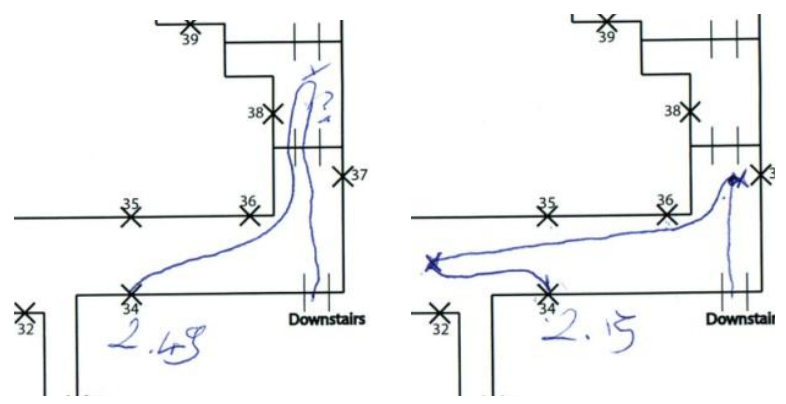

Figure 10. Typical paper map participant behavior (emerging from staircase and going straight ahead before realizing the target is to the left).

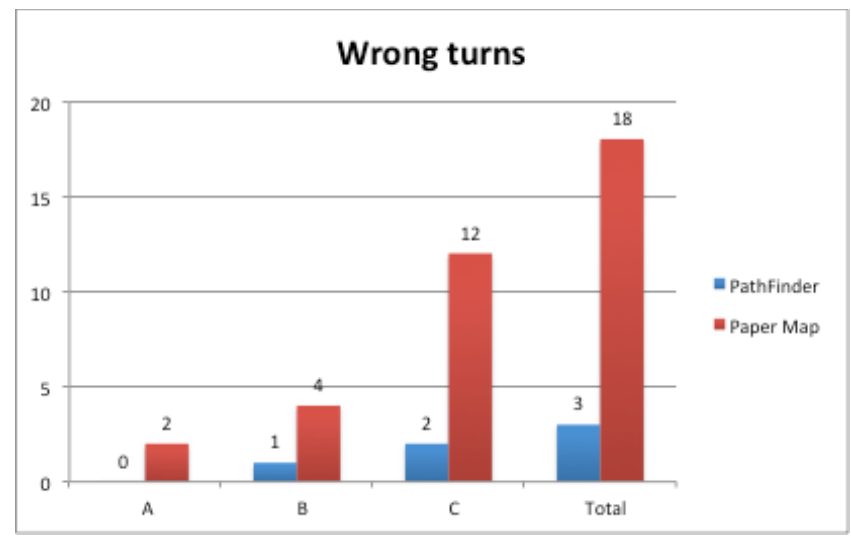

Figure 11. Wrong turn performance

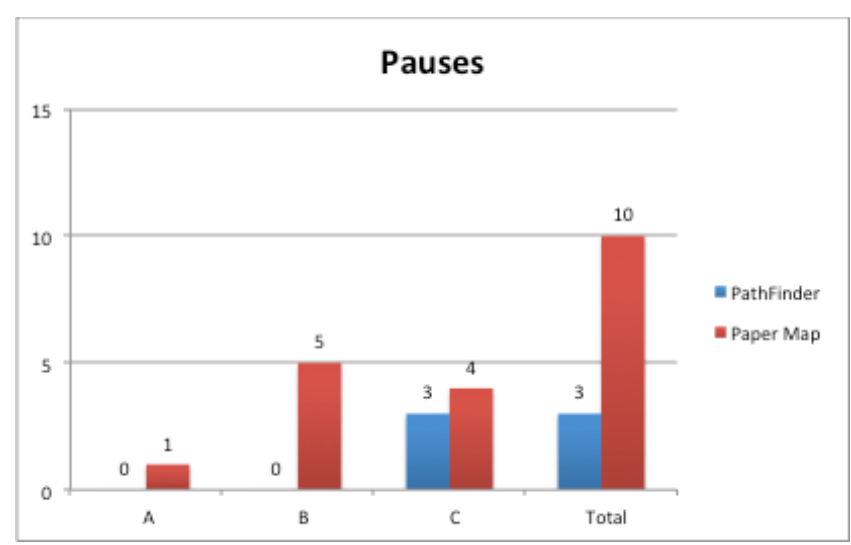

Figure 12. Pause performance

\section{Statistical Analysis of Observations}

Table 3 shows the statistical analysis for the correlation between task completion times and the number of wrong turns and pauses for Groups 1 and 2. For Group 1, we also show the relevant analysis for scan failures. Where ' $\mathrm{n} / \mathrm{a}$ ' is shown, there were no observations for any participants for the type of event.

Though statistical significance cannot be claimed for some of the measurements (particularly Group 1), it appears that for Group 1 there is a relationship between the scan failures and the total time taken to complete the experiment, which borders on being significant. On the other hand, in Group 2, it is clear that the higher number of 'error' events during task completion had a statistically significant impact on the time taken to complete the experiment. Two important conclusions can thus be derived from these findings. Firstly, an indoor navigation application has to keep users moving. As seen on both occasions, pausing in order to re-orient has a significant effect on the time it takes a user to complete a task. Additionally, it is clear that additional support should be offered to users when the application detects that they have strayed off-target. Currently our system does not offer this, but a mini- map showing the user's current position and target position might help the user to quickly re-orient and move without needing to scan too often. 
TABLE III. CORRELATION OF TASK COMPLETION TIME AND OTHER VARIABLES FOR GROUPS 1 AND 2

\begin{tabular}{|c|c|c|c|}
\hline \multicolumn{4}{|c|}{ Group 1 R(9) } \\
\hline & Wrong turns & Pauses & Scan Failures \\
\hline Task A & $\mathrm{n} / \mathrm{a}$ & $\mathrm{n} / \mathrm{a}$ & $0.647, p=0.59$ \\
\hline Task B & $0.24, p=0.538$ & $\mathrm{n} / \mathrm{a}$ & $0.188, \mathrm{p}=0.628$ \\
\hline Task C & $0.75, \mathrm{p}<0.05$ & $0.855, \mathrm{p}<0.01$ & $0.070, p=0.857$ \\
\hline Total & $0.09, \mathrm{p}=0.815$ & $-0.102 \mathrm{p}<0.01$ & $0.552, \mathrm{p}=0.123$ \\
\hline \multicolumn{4}{|c|}{ Group 2 R(8) } \\
\hline Task A & $0.79, \mathrm{p}<0.05$ & $0.093, p=0.83$ & - \\
\hline Task B & $0.74, p<0.05$ & $0.747, \mathrm{p}<0.05$ & - \\
\hline Task C & $0.31, p=0.453$ & $0.620, \mathrm{p}<0.10$ & - \\
\hline Total & $0.73, p<0.05$ & $0.749, \mathrm{p}<0.05$ & - \\
\hline
\end{tabular}

\section{Subjective evaluations}

At the end of the trials, we asked the participants in Group 1 to subjectively evaluate their experience using our system. We asked 10 questions. 7 of the questions were quantitative, where the answer was given in a scale of 1 to 10 (higher is better). Questions 8 to 10 were open questions, which collected some qualitative data about the application. The scaled questions are show in Fig. 11.

Q1. The application helped me in getting to the destination

Q2. The application was easy to use

Q3. Arrows and directions were easy to understand

Q4. I enjoyed using this application

Q5. I find this application useful

Q6. I prefer to use this application over trying to find the shops by looking at information points and asking other people on how to get there

Q7. I find this application frustrating

Figure 13. Questionnaire questions

From the quantified answers to these questions, overall it can be seen in Fig. 12 that the application received positive feedback. The lowest score has been given to questions 3 and 6. Users gave their lowest scores to question 3, which asked them to rate how easy it was to follow the arrows and directions, indicating that there is room for improvement in the design of the AR elements in our application. Users also indicated slight concerns in relation to the question on their preference to use this application instead of regular paper maps (question vi); none of the participants decided to expand their thoughts on this within the open questions.

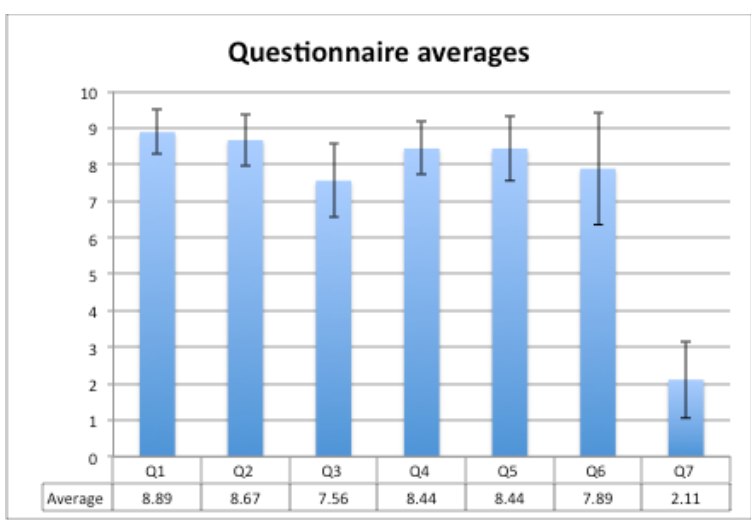

Figure 14. Questionnaire ratings
The open-ended questions asked participants to state the features they particularly liked or disliked about the prototype and also for comments for its improvement. The comments received can be summarised as follows:

- Three participants emphasised that the application was "easy to use".

- Three participants responded that they liked the use of augmented 3D arrows.

- Four of the participants expressed their disappointment with the "turnaround" arrow.

- Two participants stated that "directions were simple and easy to follow".

- One participant commented that the application was "great fun".

- Two of the participants were not happy with text directions appearing at the bottom of a screen. Participant no 2 was more specific about this problem, stating that directions did not accord with the orientation of the device screen.

- Three of the participants said that they would like voice directions added to the application, as a means of improving the application,.

- Two participants indicated that they would prefer a graphical interpretation of the directions at the bottom of the screen instead of text directions.

- One participant mentioned that he would like to be able to see the map of the building in the application.

- Participant no 2 also mentioned that she would like instructions to change in continuously, like in GPS systems.

\section{CONCLUSIONS \& FUTURE WORK}

We have described a system for navigation in indoor environments that uses features of these environments as position markers, instead of requiring special markers or other infrastructure. The use of AR to provide navigation is shown to have a clear advantage over the use of paper maps, particularly in complex navigation tasks over multiple floors. Further work on the system will examine the optimal presentation of navigation instructions as well as the use of the system in stressful conditions in complex buildings, such as emergency evacuations.

To overcome the issue of finding suitable environments for testing our system and any adaptations to the user interface, we are particularly interested in examining the utility of virtual environments with users. As a first step, we have built a complex interior environment using Unity, which has been populated with recognisable features (shop brands). A user can then stand in front of a large projected view of this environment and navigate through it, using our AR system to recognise features (Fig. 13). Without any modification to the targets used in our original trial, early validation tests seem to indicate that the virtual environment can be successfully used in order to test complex scenarios in computer models of actual indoor environments, prior to attempting a field trial in the physical settings (Fig. 14). 
We are in the process of investigating suitable interaction modalities for the participants, which will enable them to naturally navigate the virtual environment and use a handheld AR device at the same time. We are, at the moment, taking a simple approach by combining a gaming console joypad with a mobile device. This will allow the user to hold the interaction device at a lowered position to control walking and view angle through analog thumb joysticks and raise the device to use its mobile AR part near a trackable feature. Though simplistic, we feel this interaction modality might prove usable. We are, however, aiming to investigate more advanced modalities such as whole body interaction with the environment through wearable sensors, which we have used with success in the past [13].

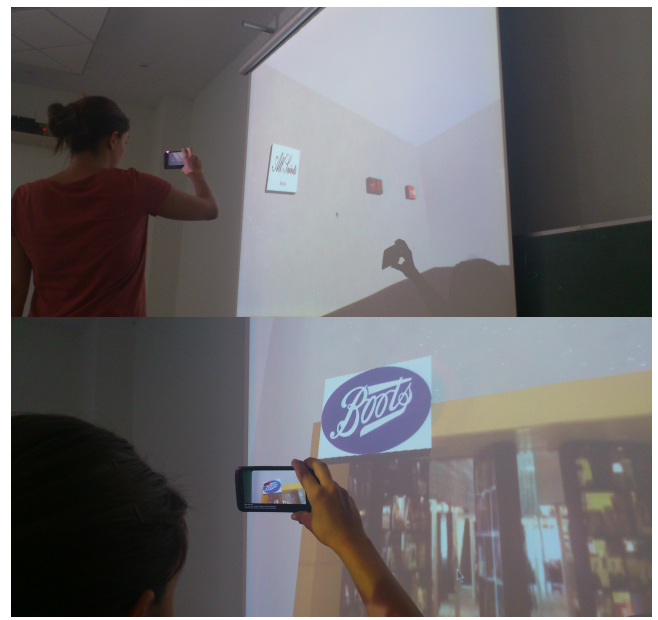

Figure 15. The synthetic (virtual) environment in use with the Pathfinder system

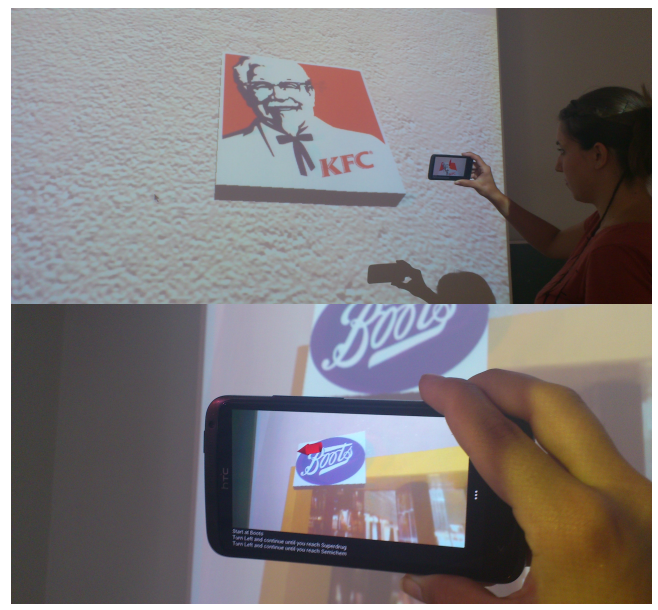

Figure 16. Scanning indoor features in a virtual environment. The navigation instructions and directional arrow are visible in the lower image.

\section{REFERENCES}

[1] T.M.T. Do, J. Blom and D. Gatica-Perez "Smartphone usage in the wild: a large-scale analysis of applications and context" In Proceedings of ICMI. 2011, 353-360, 2011

[2] A.J.B. Brush, A.K. Karlson, J. Scott, R. Sarin, A. Jacobs, B. Bond, O. Murillo, G.C. Hunt, M. Sinclair, K. Hammil and S. Levi "User experiences with activity-based navigation on mobile devices". In Proc. MobileHCI 2010, pp. 73-82, 2010.

[3] L. Chittaro and D. Nadalutti "Presenting evacuation instructions on mobile devices by means of location aware 3D virtual environments", In Proc. MobileHCI 2008, pp. 395-398, 2008

[4] K. Kaemarungsi and P. Krishnamurthy "Modeling of indoor positioning systems based on location fingerprinting", In Proc. INFOCOM 2004 vol.2, no., pp. 1012-1022, 2004

[5] M. Klopschitz and D. Schmalstieg "Automatic Reconstruction of Wide-Area Fiducial Marker Models”, In Proc. ISMAR 2007. pp.7174, 2007

[6] D. Merico and R. Bisiani "Indoor Navigation with Minimal Infrastructure". In Proc 4th WPNC 2007, pp. 141- 144, 2007

[7] A. Mulloni, H. Seichter and D. Schmalstieg "Handheld Augmented Reality Indoor Navigation with Activity-Based Instructions", In Proc. MobileHCI 2011, 20011

[8] A. Mulloni, D. Wagner, I. Barakonyi and D. Schmalstieg "Indoor Positioning and Navigation with Camera Phones", IEEE Pervasive Computing, vol.8, no.2, pp.22-31, 2009

[9] G. Reitmayr and D. Schmalstieg, "Location based applications for mobile augmented reality". In Proc OzCHI 2003 pp. 65-73, 2003

[10] J. Kim and H. Jun "Vision-based location positioning using augmented reality for indoor navigation", IEEE Transactions on Consumer Electronics, vol.54, no.3, pp.954-962, 2008

[11] D. Wagner and D. Schmalstieg, "First steps towards handheld augmented reality". In Proc. 7th IEEE International Symposium on Wearable Computers, 127-135, 2003

[12] H.J. Müller and J. Schöning, A. Krüger "Mobile Map Interaction Evaluation in an indoor scenario". GI Jahrestagung (2)’06, (2006), 403-410

[13] P. Barrie, A. Komninos, and O. Mandrychenko. "A pervasive gesturedriven augmented reality prototype using wireless sensor body area networks." In Proceedings of the 6th International Conference on Mobile Technology, Application \& Systems, p. 61. ACM, 2009.

[14] D. Robertson and R. Cipolla "An image-based system for urban navigation", In The 15th British Machine Vision Conference (BMVC'04), Kingston-upon-Thames, UK pp. 819-828, 2004

[15] K. Tollmar, T. Yeh and T. Darrell "IDeixis - Searching the Web with Mobile Images for Location-Based Information" In Proc. MobileHCI 2004. pp. 61-125, 2004

[16] F. Cavallo, A. M. Sabatini and V. Genovese "A step toward GPS/INS personal navigation systems: real-time assessment of gait by foot inertial sensing". In 2005 IEEE/RSJ International Conference on Intelligent Robots and Systems, pp. 1187 - 1191, 2005

[17] Z. Liu, C. Aduba and C.H Won "In-plane dead reckoning with knee and waist attached gyroscopes". Measurement, 44(10), pp.1860$1868,2011$.

[18] C. Kray, C. Elting, K. Laakso and V. Coors "Presenting route instructions on mobile devices", In Proc. of the 8th international conference on Intelligent user interfaces. IUI '03. New York, NY, USA: ACM, pp. 117-124, 2003. 